\title{
Ultrasonic processing of molten and solidifying aluminium alloys: overview and outlook.
}

\author{
Dmitry Eskin 1,2 a \\ ${ }^{1}$ Brunel University London, BCAST, Uxbridge UB8 3PH, United Kingdom \\ ${ }^{2}$ Tomsk State University, Tomsk, 634050 Russia \\ admitry.eskin@brunel.ac.uk
}

Keywords: Aluminium; Cavitation; Composite; Degassing; Grain refinement; Solidification; Ultrasound

\begin{abstract}
Ultrasonic melt processing attracts a lot of interest both from academic researchers and industry. Despite a long history of the subject, with first accounts dating back to the 1930s, the physics, mechanisms and practical applications are still under development. In the second half of the XXth century, pilot- and industrial scale applications of ultrasonic processing of light alloys were demonstrated for melt degassing, filtration, grain refinement, melt atomisation, and zone refining. In the last 10 years the interest to ultrasonic melt processing grew with regard to understanding the underlying mechanisms of previously established effects, developing numerical models of ultrasonic cavitation and the development of nanocomposite technology. This review paper summarises the mechanisms involved in the ultrasonic melt processing, including cavitation, flows, nucleation, activation, fragmentation and their consequences for degassing, structure refinement and particle dispersion. Some typical mistakes made by researchers in performing experiments and in interpretation of the results are discussed. New advanced methods of studying ultrasonic treatment and phenomena are considered. The paper also gives an outlook to future developments and challenges.
\end{abstract}

\section{Introduction}

Ultrasonic melt treatment is known for at least 60 years as a potentially efficient, clean and versatile technology that can be used for melt processing (degassing, cleaning, filtration), solidification processing (grain refinement) and materials manufacturing (composite materials) $[1,2,3,4,5]$. At the same time, the ultrasonic melt processing is still mostly confined to laboratory.

The main fundamental mechanisms of ultrasonic melt processing have been developed in the 1950s-1970s and remain virtually unchanged since. New developments are mostly related to computer modelling and process simulations, use of sophisticated experimental techniques for characterization as well as to the application of more advanced ultrasonic equipment that makes the upscaling of the technology easier.

Among the reasons for limited industrial application are misconceptions and incorrect interpretation of results that hinder the up-scaling as well as the lack of adequate multiscale models that can be used in process simulation.

The basis of ultrasonic processing is the formation of cavitation bubbles in the melt subjected to high-frequency, high-power vibrations, their pulsation and collapse in the acoustic field, and acoustic streaming that extends the action of ultrasound to a larger melt volume. Figure 1 illustrates the main regions of ultrasonic processing in the liquid phase.

The main technological areas of ultrasonic processing are melt degassing, grain and structure refinement and production of composites [6].

In recent years, the interest to ultrasonic melt processing is growing due to various reasons. In some cases, like degassing, the environment-friendly, zero-emission and energy-efficient ultrasonic technology attracts attention of both foundries and cast houses. For grain refinement, the possibility of achieving small grain size and fine primary particles without special additions or at significantly 
reduced levels of these additions is attractive from both economic and technological viewpoints. Manufacturing metal-matrix composite materials with nano-sized reinforcement through a liquidmetal route seems impossible without application of radical external fields, including ultrasonic cavitation. Also new means of studying the cavitation became available in recent years, including in-situ studies using synchrotron radiation, direct measurements of acoustic spectrum and pressure using a cavitometer, and sophisticated multi-scale, multi-physics numerical models.

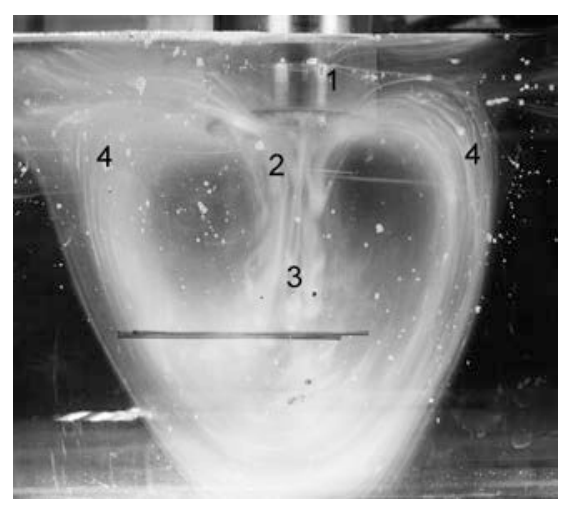

Figure 1. Typical zones in ultrasonic processing of a liquid phase: 1 , sonotrode; 2 , cavitation zone; 3 , acoustic stream; and 4, recirculation flow. Filmed in glycerine using a 1-kW transducer with $\mathrm{Ti}$ sonotrode at $20 \mathrm{kHz}$. Courtesy of $\mathrm{I}$. Tzanakis.

\section{Main Mechanisms and Applications of Ultrasonic Melt Processing}

Ultrasonic Degassing. Ultrasonic degassing is based on the principle of rectified diffusion of hydrogen from the liquid aluminium into a bubble pulsating in the acoustic field [7]. The oscillating bubbles act like small pumps extracting dissolved hydrogen from the melt. Eventually the bubbles grow and float to the surface, releasing the hydrogen to the atmosphere.

Hydrogen finds its way into liquid aluminium mostly from atmospheric humidity. Water vapor reacts with liquid aluminium to produce aluminium oxide and hydrogen. As the partial pressure of hydrogen is very high, liquid aluminium, in principle, can dissolve almost any amount of hydrogen. The actual quasi-equilibrium solubility of hydrogen is a function of humidity, temperature and pressure and varies from day to day, and from alloy to alloy. In most cases the concentration of hydrogen in liquid aluminium ranges from 0.2 to $0.8 \mathrm{~cm}^{3} / 100 \mathrm{~g}$, while the desired concentration for producing sound castings is about $0.1 \mathrm{~cm}^{3} / 100 \mathrm{~g}$. Ultrasonic degassing is capable of decreasing the amount of dissolved hydrogen to the levels $40-50 \%$ below the quasi-equilibrium concentration [3, 8]. It is important to note that such a low hydrogen concentration cannot be sustained for long and the melt will absorb hydrogen from atmosphere back to the quasi-equilibrium level. This process is called re-gassing and occurs rather quickly in a matter of tens of minutes [8].

Ultrasonic degassing of liquid aluminium includes two main stages: producing of cavitation bubbles that are filled with hydrogen (degassing phase) and flotation of these bubble to the surface (resting phase). Therefore, the technology should allow for the release of the bubbles. It was shown that intermittent ultrasonication with idle intervals is more efficient than the continuous ultrasonic degassing, and can achieve 90\% decrease in hydrogen concentration (degassing efficiency) in relatively small volumes $[2,8]$.

The efficiency and kinetics of ultrasonic degassing depend on the intensity of ultrasonication (acoustic power introduced into the melt, cavitation development and extent), position and shape of the sonotrode system (extent of cavitation region), melt temperature (hydrogen solubility and melt viscosity), alloy type (hydrogen solubility and oxide film structure), humidity (hydrogen solubility), and the treated volume [6].

The ultrasonic degassing can be performed as a batch operation (in a ladle or special degassing vessel) or continuously. The former is suitable for foundries, the latter is the only option for directchill casting.

In the first industrial applications of ultrasonic degassing, the problem of up-scaling to treating large melt volumes was solved by multiplication of ultrasonic sources. Up to 11 ultrasonic systems 
(transducers with sonotrodes) were deployed in a crucible or in a launder to achieve good degassing efficiency [2, 3]. This scheme, though producing the required degree of degassing, suffers from complexity and is not very economical.

In recent years other technological ideas were suggested and tested. In the batch operation the introduction of several sonotrodes was replaced with a single sonotrode moving across the surface of the melt. In this case the sonicated volume has time for the bubble release while the active phase of ultrasonic degassing happens in the other part of the treated volume. This scheme demonstrated successful degassing of a substantial volume of $150 \mathrm{~kg}$ of A356 melt with the degassing efficiency more than 30\% achieved in 15 min [8, 9]. Similar degassing efficiency was shown by the standard Ar rotary degassing (Foseco Degassing Unit) in the same volume and time. There is one important advantage of ultrasonic degassing that was revealed by pilot-scale foundry trials. The amount of dross generated at the surface of the degassed melt is 5 times less in the case of ultrasonic processing as compared to Ar-assisted degassing [9]. The reason for that is the degree of turbulence in the melt that is much greater in rotary degassing.

There are limitations, however, in how large a volume can be processed with ultrasound using a single ultrasonic source in the batch operation. Industrial conditions require degassing of hundreds $\mathrm{kg}$ of melt in a relatively short period of time or even in a continuous fashion. An obvious solution is to perform degassing in the melt flow, e.g. during the melt transfer from a melting furnace to a ladle or to a mould. A different scheme of sonication was suggested with a plate sonotrode placed inside the melt volume, e.g. close to the bottom of a launder, Fig. 2a. In this case a relatively long and thin plate oscillates in the flexural mode (a conventional sonotrode oscillates longitudinally) with much shorter wavelength, which allows for several antinode points with the maximum vibrational amplitude where the cavitation condition are met in the surrounding liquid [10]. The cavitation bubbles are formed in the adjacent melt volumes and experience rather long life while oscillating in the acoustic field. The bubbles filed with hydrogen travel downstream with the melt and are gradually released to the atmosphere. The real-time cavitation measurements performed using a state-of-the-art high-temperature cavitometer confirmed the cavitation conditions in the melt as illustrated by the frequency spectrum in Fig. 2b. Lab-scale experiments demonstrate that ultrasonic degassing with the plate sonotrode is $50 \%$ more efficient than with the conventional sonotrode in the batch operation and allows one to achieve 50\% degassing efficiency when the degassing is performed in the melt flow.

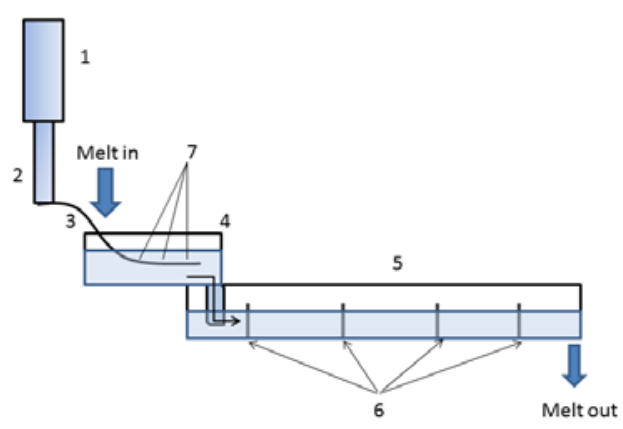

a

Figure 2. Characterization of a plate sonotrode for ultrasonic degassing in melt flow: (a) a scheme of in-line degassing with a plate sonotrode (1, transducer; 2 , transition waveguide; 3 , plate sonotrode; 4, degassing chamber; 5, launder; 6, baffles; 7, points of cavitation generation) and (b) frequency spectrum of acoustic noise measured in liquid aluminium by a high-temperature cavitometer indicating high-frequency signals from collapsing and pulsating bubbles (adapted from [10]).

The current research is focused on process modelling of ultrasonic degassing with main challenges related to the interaction of the cavitation zone with the macroscopic melt flow and to the extent of the acoustic field interaction with cavitation and gas-filled bubbles [11]. 
Structure Refinement. Grain refinement as well as refinement of primary particles such as Si and intermetallics is a well-known effect of ultrasonic processing. There are a number of mechanisms suggested to explain the refining effect of ultrasonic cavitation, these are reviewed elsewhere [3, $6,12]$. It is important to understand that these mechanisms seldom work simultaneously and are specific for certain temperature ranges.

A cavitation bubble can facilitate nucleation of a solid phase through changing the local equilibrium conditions upon bubble implosion. The extremely high pressure generated upon bubble collapse (in the range of GPa) increases the solidification temperature so that the liquid phase become greatly undercooled and may form stable nucleus [13]. The survival of the nucleus is subject to the melt temperature and flow. This mechanism, though theoretically possible for homogeneous nucleation, may be more applicable to the conditions of heterogeneous nucleation when occurring close to the liquidus.

On the other hand, this mechanism can combine with the so-called substrate activation. The activation means that an inert particle floating in the melt (e.g. oxide, carbide, boride) becomes an active substrate through the physical action of cavitation. There are several phenomena that may participate in the activation. Firstly the melt needs to gain access to the surface of the particle. In many cases the surface of the particle in the molten aluminium is "coated" with absorbed hydrogen and the surface defects of the particles are filled with molecular hydrogen, which prevents the access of the melt to the surface of the particle. Cavitation bubbles pulsate and collapse producing powerful jets and surges of high pressure and temperature, especially close to the interfaces. As a result the particles are stripped of the absorbed gas and the surface defects are filled with liquid aluminium (sono-capillary effect) [3]. If the particle represents a compound that has low surface tension with liquid aluminium then the particle become wetted and may act as a solidification substrate (cavitation also eases the nucleation at this wet surface, see the previous paragraph). If the compound is not wetted well with the liquid aluminium, the particle can still be active due to the melt penetrating its surface defects. Because of the high capillary pressure, this melt can solidify and remain solid at ambient temperatures above the liquidus. Hence, when such a particle finds itself below the liquidus temperature, the solidification starts on the pre-existing patches of solid aluminium. Figure 3 demonstrates the nucleation of the $\mathrm{Al}_{3} \mathrm{Zr}$ phase on an alumina particle and refinement of this phase with ultrasonic processing.

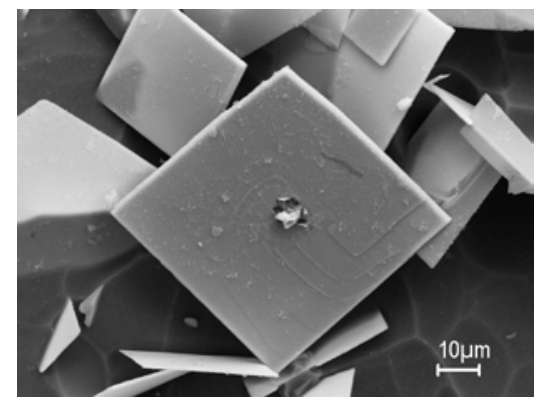

a

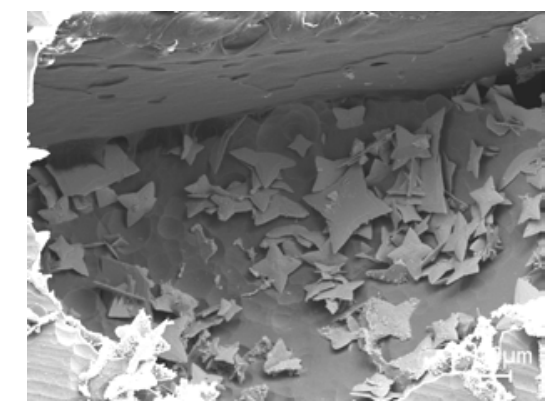

b

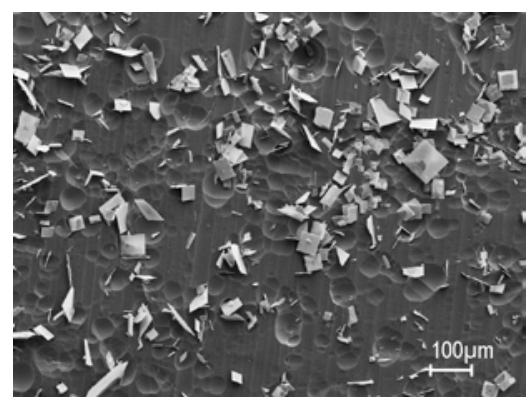

C

Figure 3. Effect of ultrasound on the formation of primary $\mathrm{Al}_{3} \mathrm{Zr}$ intermetallic in an $\mathrm{Al}-0.4 \% \mathrm{Zr}$ alloy: a, nucleation on an $\mathrm{Al}_{2} \mathrm{O}_{3}$ particle (seen in the centre of the intermetallic); primary intermetallics formed without ultrasonic processing (b) and with ultrasonic processing (c). Deep etching. Courtesy of F. Wang.

The other very powerful mechanism of refinement is fragmentation of primary crystals and dendrites. It is not entirely clear how the fragmentation happens. Very limited direct observations show a mixture of rapid fragmentation from collapsing bubbles, slow separation of fragments assisted by pulsating bubbles and detachment of dendrites from the solidification front assisted by acoustic flow [14, 15, 16]. Most likely a combination of solute, flow and momentum effects plays role in the fragmentation. For primary $\mathrm{Al}$ grains the ultrasonic treatment performed during 
solidification always results in significant grain refinement (Fig. 4). However, this effect is always confined to relatively small volumes where the cavitation and acoustic streaming may be still active under conditions of progressively increasing volume fraction of solid accompanied by greater viscosity and sound wave attenuation.

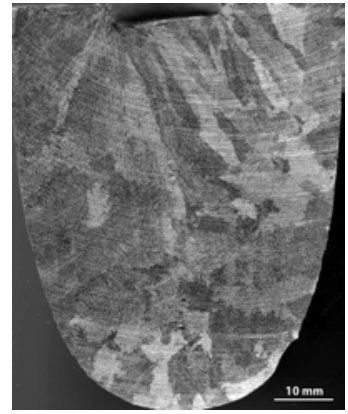

a

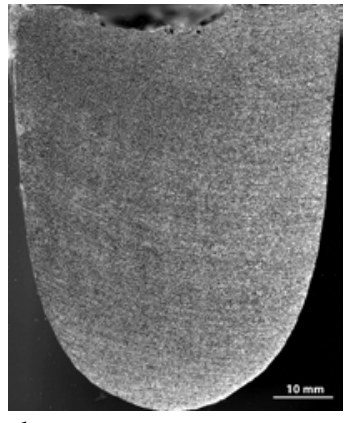

b
Figure 4. Effect of ultrasonic processing during solidification of an Al-4\% Cu alloy: a, no US, submerged idle sonotrode and b, US $(17.5 \mathrm{kHz}$, $40 \mu \mathrm{m}, \mathrm{Nb}$ sonotrode) from 700 to $580{ }^{\circ} \mathrm{C}$ (adapted from [17]).

For the best effect, the ultrasonic processing should be applied continuously from above the liquidus through the upper part of the solidification range of the phase that should be refined. This was confirmed for primary intermetallics [17] and Si [18] as well as for primary aluminium dendrites [19]. Isothermal ultrasonic treatment in the semi-solid state results rather in grain coarsening [20]. This effect is most likely to be a result of limited cavitation and flow extent in the mushy semi-solid environment, while the thermal energy produced by cavitation causes re-heating of the semi-solid material with ensuing coarsening.

The final refined structure may be a result of several mechanisms acting sequentially, e.g. in the case of grain refining of aluminium alloys with $\mathrm{Zr}$ and $\mathrm{Ti}$. The ultrasonic processing starts at a temperature above the formation temperature of $\mathrm{Al}_{3} \mathrm{Zr}$. Activation of some inclusions facilitates nucleation of the primary intermetallics (se Fig. 3). On decreasing the temperature below the formation of temperature of $\mathrm{Al}_{3} \mathrm{Zr}$ (e.g. $720-740{ }^{\circ} \mathrm{C}$, depending on $\mathrm{Zr}$ concentration) the particles nucleate and also become fragmented so that their number density rapidly increases. Further down the solidification range on reaching the liquidus of the aluminium solid solution, aluminium start to nucleate on fine and numerous $\mathrm{Al}_{3} \mathrm{Zr}$ particles. If ultrasonic process continues below $660{ }^{\circ} \mathrm{C}$ some additional fragmentation of growing dendrites occurs. The ultrasonic processing should be stopped while the alloy is still fluid (e.g. at temperatures within $5^{\circ} \mathrm{C}$ below the Al liquidus). Ti plays role as a growth restriction element and further refines the grain structure. The efficiency of this approach has been confirmed of different alloying systems [17, 21], Fig. 5.

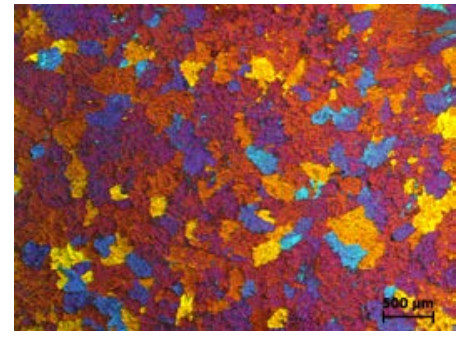

a

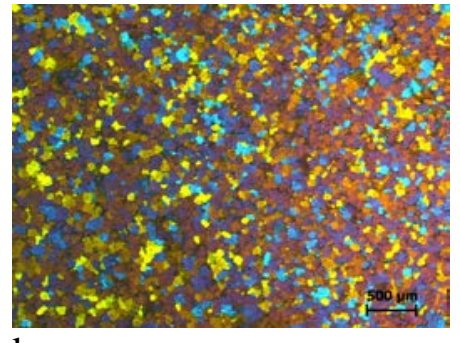

$\mathrm{b}$

Figure 5. Effect of ultrasonic melt processing on the grain refinement in an $\mathrm{Al}-2 \% \quad \mathrm{Ni}-1 \% \quad \mathrm{Mn}-0.2 \% \quad \mathrm{Zr}-0.08 \% \quad \mathrm{Ti}$ alloy: (a) no ultrasonic processing (grain size $230 \mu \mathrm{m}$ ) and (b) with ultrasonic processing (grain size $72 \mu \mathrm{m}$ ).

Under industrial conditions, the structure refinement using ultrasonic processing can be performed in the launder close to the mould or in the liquid sump of a billet during DC casting [6] or in the feeding/gating system of a shape casting.

Dispersion and De-agglomeration. One of the reasons for the increased interest to ultrasonic processing is the potential of acoustic cavitation to break agglomerates of small particles and the ability of acoustic streaming to disperse the particles inside the melt volume. In addition, the improved wettability of nonmetallic particles facilitates their distribution and bonding with the 
metallic matrix. These phenomena are very useful in manufacturing master alloys with nonmetallic particles (e.g. $\mathrm{TiB}_{2}$ ) and composite materials with small, even nano-sized, reinforcing particles.

The application of ultrasonic cavitation to making metal-matrix composites was actually suggested long ago and some positive results have been presented elsewhere [3, 22].

Theoretically the pressure generated by collapsing cavitation bubbles should be enough to break agglomerates of nanoparticles [23]. In reality a combination of poor wettability, surface contamination of the particles and pushing of the particles during solidification makes it difficult to achieve homogeneous distribution of reinforcement throughout the solid phase. Most of the particles are located at dendrite boundaries, frequently associated with eutectic phases and porosity. Nevertheless, the ultrasonic processing greatly improves the distribution of particles as shown in Fig. 6a, b.

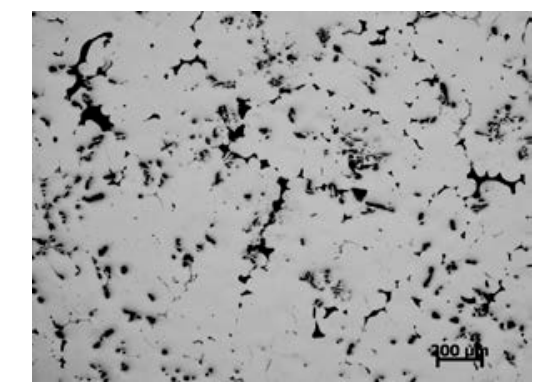

a

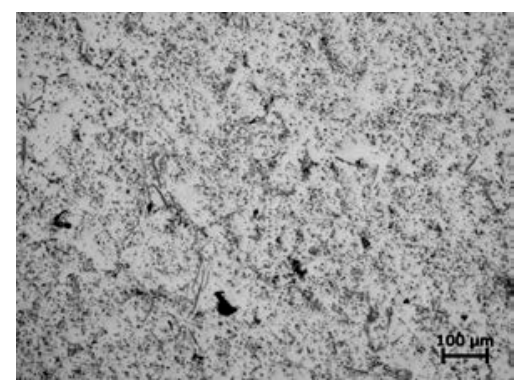

b

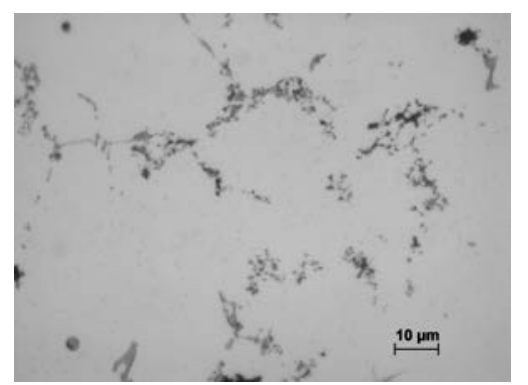

C

Figure 6. Composite materials: (a) $\mathrm{Al}-2 \mathrm{wt} \% \mathrm{TiB}_{2}$, stirring; (b) $\mathrm{Al}-2 \mathrm{wt} \% \mathrm{TiB}_{2}$, stirring and ultrasonic processing; (c) Al-0.5\% nAlN produced using the master alloy and ultrasonic processing. Courtesy of S.K. VadakkeMadam, J. Tamayo and S.A. Vorozhtsov.

The way how the particles are introduced into the melt plays its role as well. Usually, a loose compact of particles is made, wrapped in single- or double-layer aluminium foil and introduced into the melt with mechanical stirring simultaneously or sequentially with ultrasonic melt processing. In some cases, a protective atmosphere is used to reduce the surface oxidation of the melt. Relatively small volumes (up to $1 \mathrm{~kg} \mathrm{Al}$ ) require long processing times (up to $1 \mathrm{~h}$ ) to achieve sufficient distribution of particles with some rejected particles remaining. A more suitable way of particle introduction is through a pre-mixed concentrated master alloy. There are different techniques to produce such a master alloy from powders of aluminium and nanoparticles, e.g. extrusion, microwave sintering, and detonation. A master alloy produced by detonation is characterized by uniform structure, high density and goo bonding between nanoparticles and the matrix [24]. Such a master alloy in a form of a rod can be easily introduced into the cavitation zone under the sonotrode. Preliminary experiments showed good dissolution of the master alloy without particle rejection. The nanoparticles are uniformly distributed in the casting volume (Fig. 6c). The degree of their distribution in the microscopic level (within a grain or group of grains) depends on the processed volume and time of ultrasonic processing, but the time required is considerably shorter than in the case when loose particles are introduced.

\section{Recent Advances in Characterisation}

Characterisation of ultrasonic processing (cavitation, streaming, etc.) have been confined until recently to rapid filming of transparent liquids (see Fig. 1) [14, 15] and to a qualitative analysis of acoustic spectrum of cavitation noise [3]. The development of in-situ characterisation using highintensity X-ray produced in synchrotron enables direct observations of cavitation bubbles in liquid metals [16, 25, 26] (Fig. 7) and their interaction with inclusions [27]. At the same time, new generation cavitometers are able to measure acoustic spectrum (see Fig. 2b) and, if calibrated, acoustic pressure in liquids, including molten metals [10, 28]. All these advances have already 
contributed and continue to contribute to our understanding of the phenomena occurring during cavitation melt processing as well as provide essential data for validation of numerical models.
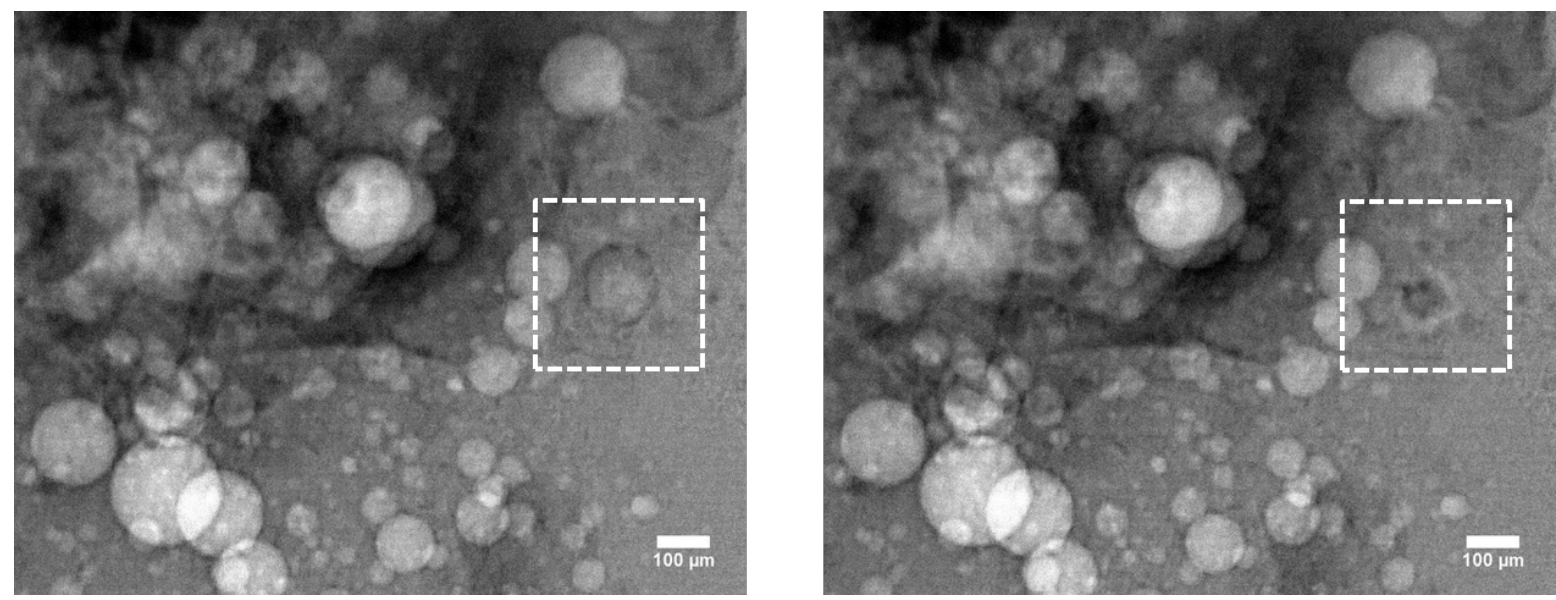

Figure 7. Direct observation of cavitation bubble collapse (framed) upon ultrasonic processing of a liquid aluminium alloy. Courtesy W.W. Xu, I. Tzanakis, W. Mirihanage, P. Srirangam, P.D. Lee.

\section{Typical Mistakes and Misconceptions}

Selection of Equipment and Materials. Most of current laboratory experiments with liquid metals are performed using widely available piezoceramic transducers. The advantages of these transducers, apart from their availability from a selection of producers, are high energy efficiency, wider range of available frequencies, controlled amplitude and frequency under load, and compactness. The core of these transducers is a stack of ceramic crystals that contract under alternating electrical field. The other type of transducers is based on magnetostrictive principle when a stack of magneto-sensitive metallic sheets contract and expand in alternating electromagnetic field. Magnetostrictive transducers are mostly used at low ultrasonic frequency, have relatively low energy efficiency and require water cooling.

The advantages of piezoceramic transducers may turn to disadvantages when high-temperature applications are concerned. This is related to the heating protection of the transducer and to the ability of frequency tuning under changing resonance conditions as a result of expansion and changing acoustic properties of the heated sonotrode.

An advantage of magnetostrictive transducers for metallurgical applications is their water cooling that prevents the transducer from overheating, maintaining the temperature below the Curie point even when the working sonotrode is dipped into the molten metal for a considerable period of time. Yet another advantage over piezoceramics is in recovery of magnetostrictive properties in the case of overheating above the Curie point. The recovery requires just cooling of the magnetostrictive stack, while piezoceramics would need ex-situ re-polarization. During melt processing the sonotrode exposed to high temperatures expands and changes its dimensions, deviating for resonance length. This does not pose a huge challenge for magnetostrictive systems that can tune to a new resonance conditions by adjusting the frequency in a relatively wide range. Most of piezoceramic systems have very narrow margin of tuning and, being incapable of maintaining the set amplitude, switch off or fracture due to overload.

As a result the experiments performed using piezoceramic-based equipment are necessarily confined to small volumes and short processing times. The use of magnetostrictive equipment allows one to extend treatment to hours as well as to process liquid metals at high temperatures and in large volumes. These temperatures and volumes will be limited by the sonotrode material and the localization of cavitation and flow phenomena, but not by equipment. In fact the only known industrial-scale applications of ultrasonic melt processing technology - up to $200 \mathrm{~kg}$ of aluminium 
melt in a crucible or up to 1 ton in the melt flow upon direct-chill casting of large ingots - have been achieved using one or several magnetostrictive transducers working simultaneously or in sequence [2, 3].

The selection of material for a sonotrode (horn, tip) is one of the very important practical considerations. The sonotrode dipped into the molten metal and exposed to high-frequency vibrations and cavitation dissolves, erodes and fractures at a scale much larger than the same piece of metal in the quiet melt. The choice of the material should be made with taking into account its stability under cavitation conditions in the melt. Although this seems obvious, many experiments are still performed using steel or titanium that are not suitable either for continuous operation in aluminium melts or for studying structure modification. It has been determined back in the 1960s that steel dissolves and erodes very quickly (in a matter of minutes) in liquid aluminium contaminating it and changing its composition; while titanium dissolves slower but as was recently shown has clear effect on the grain size acting as growth restriction element $[2,3,29]$. The effect of dissolved Ti (from a sonotrode) is clearly shown in Fig. 8. The increase in Ti concentration in the melt was from 0.002 to $0.02 \%$ after $15-30$-s cavitation exposure at $730^{\circ} \mathrm{C}$. As a result, the grain refinement occurred that was not related to ultrasonic processing as demonstrated by the structure of the same alloys processed using a Nb sonotrode. This example shows how easily one can mistake simple alloying for the effect of ultrasound.

Special experiments on the stability of metallic sonotrodes in liquid aluminium showed that $\mathrm{Nb}$ and its alloys have advantage over Fe, Ti and Mo-based materials [2, 6, 30].

The recent promising development in sonotrode materials is the use ceramics, e.g. Sialon-based [31]. Although ceramics and glass were the first choice for sonotrodes back in the 1930-1950s, then available materials such as quartz and alumina were not reliable and failed through cracking. Modern ceramics have better toughness and even can be machined to some extent. At the same time the connection of a ceramic piece to metallic parts of a waveguiding system remains a challenge and is currently done by metallic inserts into ceramics or by clamping [32].

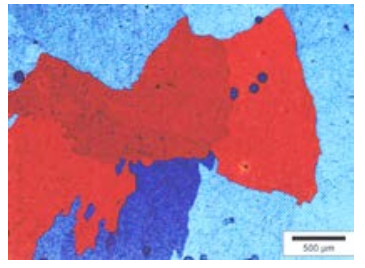

a

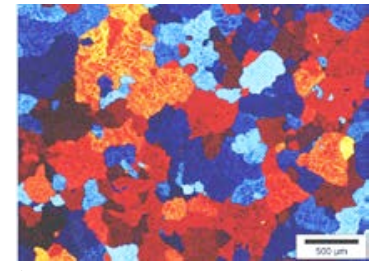

$\mathrm{b}$

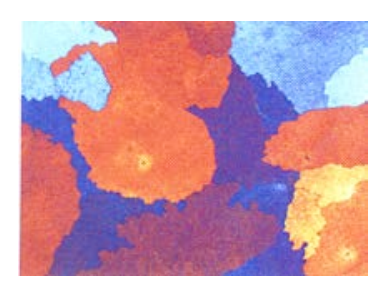

c

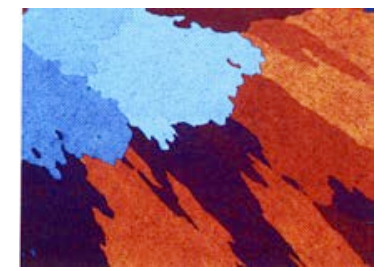

d

Figure 8. Microstructure of an $\mathrm{Al}-0.2 \% \mathrm{Zr}$ alloy treated with $\mathrm{Ti}$ (a, b) and $\mathrm{Nb}$ (c, d) sonotrodes at $730{ }^{\circ} \mathrm{C}$ : a, c, idle sonotrode immersed and b, d, working sonotrode immersed. Treatment time $15 \mathrm{~s}$, amplitude $30 \mu \mathrm{m}$ at $17.5 \mathrm{kHz}$; casting in a metallic mould. Courtesy T.V. Atamanenko.

Introduction of ultrasonic oscillations in the melt. There are two major ways to introduce the ultrasonic vibrations into the melt: direct - by insertion of the sonotrode into the melt and indirect through the mould wall or bottom.

These methods result in principally different modes of wave propagation and in different mechanisms of ultrasonic treatment. In the first method, the ultrasonic cavitation zone is formed underneath the sonotrode (providing the acoustic pressure exceeds the cavitation threshold) and the cavitating bubbles are transported by acoustic streaming to the melt, as well as forced convection pattern is established in the treated volume (see Fig. 1). The processing of the melt occurs under steady-state conditions, in the liquid state or in a pre-defined temperature range. The mechanisms of ultrasonic treatment include formation of cavitation bubbles, their oscillation and collapse with corresponding melt degassing, activation and deagglomeration of nonmetallic inclusions, and fragmentation of primary intermetallics $[3,17]$. When this scheme is used during solidification of 
the alloy, the fragmentation is responsible for the observed structure refinement. The extent of the effect in this case depends on the treated volume: small volumes can be treated completely, while in the large volumes - the effect decreases with the distance from the sonotrode, following the attenuation of the acoustic energy [33]. In the second method, the processing is done through the mould wall and the progressively solidifying shell. The acoustic conditions are constantly changing as the shell is formed and thickens. The resonance and cavitation conditions as well as acoustic contact between the sonotrode and the mould are quickly lost due to the air gap formation and the mechanical vibration results in fragmentation and fracture of solid shell, bringing about some grain refinement (Fig. 9). The grain refining effect varies with the distance from the vibrating wall. In addition progressive degassing of the melt with entrapment of gas bubbles in the solidifying metal may result in increased gas porosity as shown in Fig. 9b.

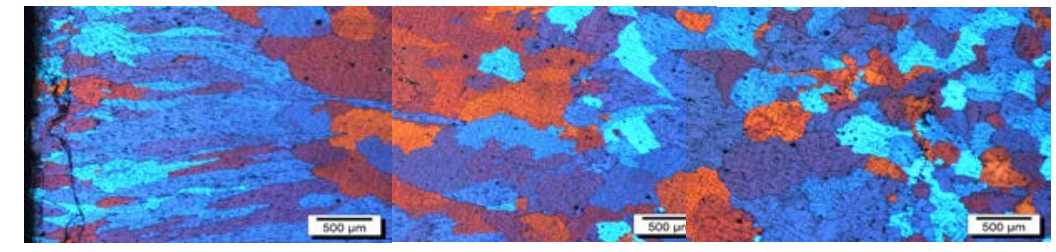

$\mathrm{a}$

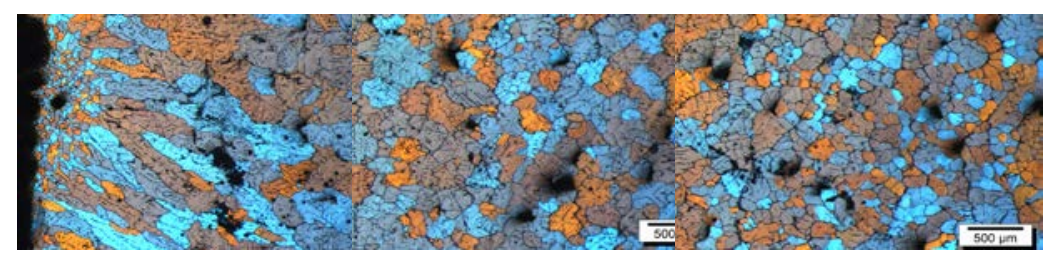

Figure 9. Structure changes

in the case when the ultrasonic vibrations are transmitted through the mould wall attached to the sonotrode (on the left): a, without ultrasound and $b$, with ultrasound, $17.5 \mathrm{kHz}$, $25 \mu \mathrm{m}$. Courtesy L. Zhang.

b

Inevitably, the ultrasonic processing performed through the walls or the bottom of the mould gives unstable results with inhomogeneous distribution of the effect through the solidifying volume. As has been already noted, the ultrasonic treatment performed during solidification is always limited to a smaller affected volume as compared to treating the liquid metal, mostly due to attenuation of acoustic energy, progressively increasing viscosity of the slurry and limited propagation of melt flows.

The optimum solution that lifts the limitations of both direct and indirect ways of ultrasound introduction, would be contactless excitation of cavitation and mixing inside the melt volume. Recent developments in electromagnetic processing may offer a solution when the high-frequency vibrations imposed on the melt by a contactless coil result in resonance conditions inside the crucible with a cavitation zone formed inside the melt volume, while low-frequency electromagnetic field induces the stirring of the melt on the macroscopic scale [34].

Cavitation mechanisms or just mixing and cooling? Some researchers try to explain the observed effects of ultrasonic processing neglecting the cavitation phenomenon and looking at seemingly simpler explanations.

In the earlier days of research in ultrasonic degassing, it was suggested that cavitation is not required for the degassing but rather the oscillation of bubbles in the acoustic field is the driving mechanism [35]. This may be true for water where there are free oxygen bubbles present and for ultrasonic-assisted Ar degassing, but the ultrasonic degassing of liquid metals surely requires cavitation as the main source of bubbles [2, 3]. Later on, there was a hypothesis that ultrasonic degassing works due to the use of Ti sonotrodes (in the 1960-1970s Ti was the material of choice for sonotrodes, as mistakenly sometimes nowadays) [36]. Titanium is a well-known getter for hydrogen so the idea was that the dissolved Ti promotes the formation of hydrides and, therefore, degassing of liquid aluminium. This misconception was rejected when efficient degassing was 
demonstrated in Ti-free aluminium melts treated using Nb sonotrodes [3]. It was also suggested that ultrasonic degassing is nothing more but natural degassing facilitated by melt agitation due to acoustic streaming [12]. This is obviously not the case as the kinetics of ultrasonic degassing and the level of residual hydrogen clearly show. Cavitation-assisted degassing is very quick (minutes) as compared with the natural degassing (tens of minutes) and the reached level is about $50 \%$ lower than the quasi-equilibrium hydrogen solubility [3, 8, 37]. The latter is evidenced by considerable regassing of ultrasonically degassed melt up to the quasi-equilibrium level [37]. In the case of natural degassing, the quasi-equilibrium level is the limit.

There is also a viewpoint that the observed effects of grain refinement in metallic alloys upon ultrasonic treatment is not related to cavitation but rather to cooling of the melt by a massive, cooled from the top metallic rod [38]. This effect of temperature drop really exists and can be very confusing when treating small volumes for a short time, like in many lab-scale experiments. Figure $10 \mathrm{a}, \mathrm{b}$ illustrates grain refinement caused by a sudden drop in melt temperature with subsequent decrease in pouring temperature and melt undercooling. It is important to note that proper experiments should involve pre-heating of the sonotrode. An additional effect of ultrasonic processing is heating of the liquid medium due to introduction of acoustic energy [3]. The increase of temperature can be substantial; and the more pronounced, the longer the processing. Therefore, during continuous operation or when the processing time exceeds several minutes, the sonotrode heats up itself and heats up the melt so there is no cooling effect anymore (Fig. 10c). Yet all effects related to cavitation, including degassing and grain refining (Fig. 10d) are still present and well reported in numerous publications.

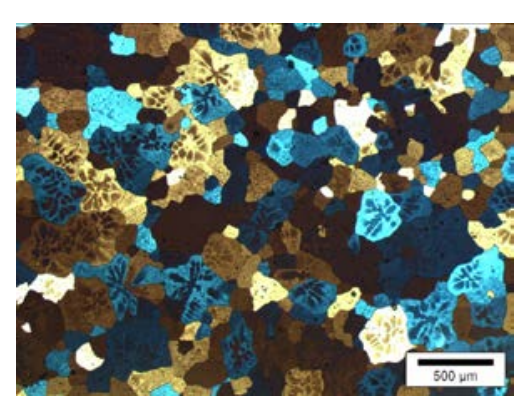

$a$

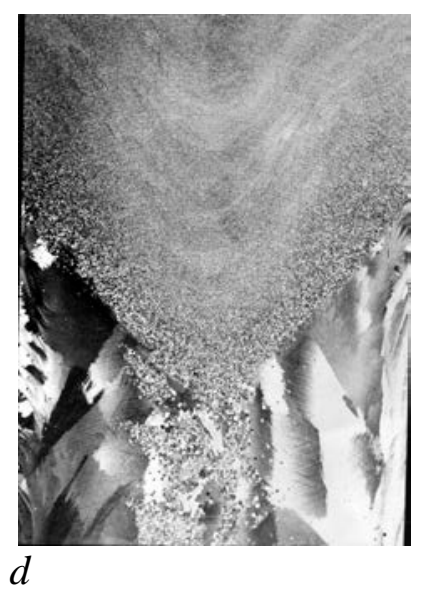

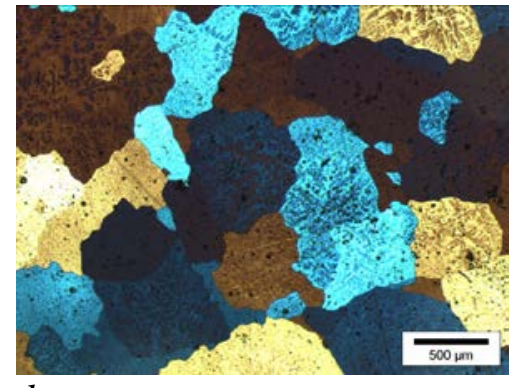

b

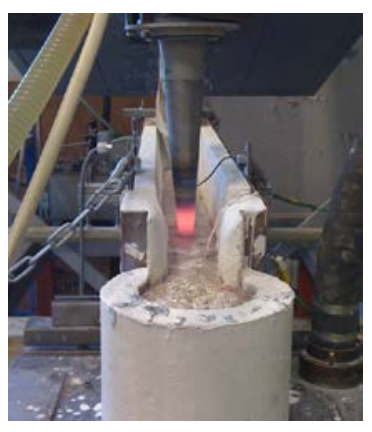

$C$

Figure 10. Effects of sonotrode temperature on structure of aluminium alloys: a, b, Al- $0.2 \% \mathrm{Zr}-0.07 \% \mathrm{Ti}$ alloy, 400 g, 15 s processing time; a, cold sonotrode and b, preheated sonotrode; c, DC casting with ultrasonic processing (note the red-hot sonotrode) and d, grain refining in an AA7050 billet with ultrasonic processing (upper part). Courtesy T.V. Atamanenko and G.I. Eskin.

\section{Summary}

Ultrasonic melt processing is a powerful and versatile means of influencing the structure and quality of cast metal. There are, however, different mechanisms and effects associated with this technology. These mechanisms are generally well known from thorough research performed since the 1950s. 
The seeming simplicity of the processing and neglecting of vast information published to date frequently lead to badly set-up experiments and wrong interpretation of the results. In this paper, some of the common misconceptions and mistakes are discussed, namely, choice of equipment, materials and temperature range of ultrasonic processing. Up-scaling of ultrasonic melt processing can be achieved by using magnetostrictive transducers, inert sonotrodes or contactless cavitation induction.

\section{Acknowledgements}

The results and data presented in this paper have been obtained within the ExoMet Project funded by the EC/FP7 (contract FP7-NMP3-LA-2012-280421); the Doshormat Project funded by the EC/FP7 (grant agreement 606090); UltraMelt project (contract number EP/K005804/1) and UltraCast project (contract number EP/L019884/1) funded by EPSRC. The author would like to thank his former and current colleagues T. Atamanenko, L. Zhang, N. Alba-Baena, K. Al-Helal, S.K. VadakkeMadam, J. Tamajo, I. Tzanakis, B. Lebon, S. Vorozhtsov, F. Wang, and Prof. G. I. Eskin for their valuable contribution to the results presented in this overview paper.

\section{References}

[1] E.A. Hiedemann: J. Acoust. Soc. Am. Vol. 26 (1954), p. 831.

[2] G.I. Eskin: Ultrasonic Treatment of Molten Aluminium (Metallurgiya, Moscow 1965).

[3] G.I. Eskin: Ultrasonic Treatment of Light Alloy Melts (Gordon and Breach OPA, Amsterdam 1998).

[4] O.V. Abramov: Crystallization of Metals in an Ultrasonic Field (Metallurgiya, Moscow 1972).

[5] H.J. von Seemann, H. Staats and K.G. Pretor: Arch. Eisenhüttenwesen. Vol. 38 (1967), p. 257.

[6] G.I. Eskin and D.G. Eskin: Ultrasonic Melt Treatment of Light Alloy Melts, Second Edn. (CRC Press, Boca Raton, FL 2014).

[7] G.I. Eskin: Ultrason. Sonochem. Vol. 2 (1995), p. 137.

[8] D. Eskin, N. Alba-Baena, T. Pabel and M. da Silva: Mater. Sci. Technol. Vol. 31 (2015), p. 79.

[9] M. da Silva, L. Rebolledo, T. Pabel, T. Petkov, X. Planta, J. Tort and D. Eskin: Int. J. Cast Metals Res. Vol. 28 (2015), p. 193.

[10] D.G. Eskin, K. Al-Helal and I. Tzanakis: J. Mater. Process. Technol. Vol. 222 (2015), p. 148.

[11] B.G.S. Lebon, K. Pericleous, I. Tzanakis and D. Eskin. Advances in the Science and Engineering of Casting Solidification, An MPND Symp. Honouring D.M. Stefanescu, (Wiley, Hoboken 2015), p. 23.

[12] J. Campbell: Int. Met. Rev. No. 2 (1981), p. 71.

[13] J.D. Hunt and K.A. Jackson: J. Appl. Phys. Vol. 37 (1966), p. 254.

[14] D. Shu, B. Sun, J. Mi and P. Grant: Metall. Mater. Trans. A. Vol. 43A (2012), p. 3755.

[15] G.M. Swallowe, J.E. Field, C.S. Rees and A. Duckworth: Acta Metall Vol. 37 (1989), p. 961.

[16] D. Tan, T.L. Lee, J.C. Khong, T. Connolley, K. Fezzaa and J. Mi: Metall. Mater. Trans. A, Vol. 46A (2015), p. 2015.

[17] T.V. Atamanenko, D.G. Eskin, L. Zhang and L. Katgerman: Metall. Mater. Trans. A. Vol. 41A (2010), p. 2056. 
[18] L. Zhang, D.G. Eskin, A. Miroux and L. Katgerman: Light Metals 2012 (Warrendale: TMS/Springer 2012), p. 999.

[19] G. Wang, M.S. Dargusch, M. Qian, D.G. Eskin and D.H. StJohn: J. Cryst. Growth. Vol. 408 (2014), p. 119.

[20] T.V. Atamanenko, D.G. Eskin and L. Katgerman: Aluminium Alloys: Their Physical and Mechanical Properties (Wiley-VCH, Weinheim 2008), p. 316.

[21] D.G. Eskin: Mater. Sci. Forum. Vols. 828-829 (2015), p.112.

[22] H.J. von Seemann and H. Staats: Z. Metallkde. Vol. 59 (1968), p. 347.

[23] H.Rumpf: Agglomeration (John Wiley, New York 1962), p. 382.

[24] S.A. Vorozhtsov, D.G. Eskin, J. Tamayo, A.B. Vorozhtsov, V.V. Promakhov, A.A. Averin and A.P. Khrustalyov: Metall. Mater. Trans. A. Vol. 46A (2015), p. 2870.

[25] W.W. Xu, I. Tzanakis, P. Srirangam, S. Terzi, W.U. Mirihanage, D.G. Eskin, R.H. Mathiesen, A.P. Horsfield and P.D. Lee: TMS Supplemental Proc., Symp. Advances in Solidification of Metallic Alloys under External Fields, ed. J. Mi, D.G. Eskin(Wiley, Hoboken 2015), p. 61.

[26] I. Tzanakis, W.W. Xu, D.G. Eskin, P.D. Lee and N. Kotsovinos: Ultrason. Sonochem. Vol. 27 (2015), p. 72.

[27] W. Mirihanage, W.W. Xu, J. Tamao, D. Eskin, M. Garcia-Fernandez, P. Srirangam and P. Lee: Scripta Mater. (2015), submitted.

[28] I. Tzanakis, G.S.B. Lebon, D.G. Eskin and K. Pericleous: Mater. Design (2015), submitted.

[29] T.V. Atamanenko: Cavitation-Aided Grain Refinement in Aluminium Alloys, PhD Thesis (Delft University of Technology, Delft 2010).

[30] S. Komarov and D. Kuznetsov: Int. J. Refract. Met. Hard Mater. Vol. 35 (2012), p. 76.

[31] S. Komarov, Y. Ishiwata and Y. Takeda: Mater. Sci. Forum • Vol .794-796 (2014), p. 124.

[32] H. Puga, J. Barbosa, J. Gabriel, E. Seabra, S. Ribeiro S and M. Prokic: J. Mater. Process. Technol. Vol. 211 (2011), p. 1026.

[33] A. Ramirez and M. Qian: Magnesium Technology 2007 (TMS, Warrenale, PA 2007), p. 127.

[34] V. Bojarevics, G.S. Djambazov and K.A. Pericleous: Metall. Mater. Trans A. Vol. 46A (2015), p. 2884.

[35] O.A. Kapustina: Physical Principles of Ultrasonic Technology (Nauka, Moscow 1970), p. 253.

[36] L.B. Maslan: Ultrasonic Treatment of Foundry Aluminium Melts (VNIIEM, Moscow 1972).

[37] N. Alba-Baena and D. Eskin: Light Metals 2013 (TMS/Wiley, Warrendale, PA 2013) p. 957.

[38] J.B. Ferguson, B.F. Schultz, K. Cho and P.K. Rohatgi: Metals. Vol. 4 (2014), p. 477. 